\title{
Studying on Non-Sampling Mixing Index
}

\author{
Migyung Cho ${ }^{1}$, Jaesool Shim ${ }^{2}$ \\ ${ }^{1}$ School of information engineering, Tongmyong University \\ 428, Sinseon-Ro, Nam-Gu, Busan, S. Korea \\ mgcho@tu.ac.kr \\ ${ }^{2}$ School of Mechanical Engineering, Yeungnam University \\ 280, Daehak-Ro, Gyeongsan, Gyeongbuk, S. Korea \\ jshim@ynu.ac.kr
}

\section{Extended Abstract}

Mixing of solid particles having different sizes, densities and shapes is very important in many industries such as the food and pharmaceutical industries. Recently, computer simulations have become an essential processing to find the optimal mixing conditions before the actual mixing task to estimate the proper level of mixing. Accordingly, there has been an increasing need for a mixing index to measure the mix of particles in the simulation process. Many mixing indices have proposed since 1950s and can largely be classified into two types: first is the statistical-based mixing index like Lacey index, Kramer index and Ashton and Valentin index[1], and the second is non-sampling mixing index using all the particles such as GMMI[2] and MGMMI[3].

In this study, we compared the two types (sampling and non-sampling) of mixing indices for the same data in the course of mixing the particles using the Discrete Element Method (DEM) simulation. For numerical simulation, total 5,000 numbers of binary particles were used to obtain mixing indices for both types. First, as pointed out in lots of articles [1,4], the Lacey, Kramer, and Ashton and Valentin indices, which are statistical based mixing indices, had different mixing index values depending on the sampling method. Second, the GMMI and MGMMI indices had high mixing index values close to 1 when the particles were completely separated into layers. They had a problem of overestimating the degree of mixing in a completely separated state. Third, GMMI and MGMMI give one or more than values as mixing index. For example, they provide five index values for a multi-component mixture with five types of particles. It can give the user a confusion about which value to choose.

With remarkable development of computer performance mixing index using the whole data will be used instead of the statistical index. However, the non-sampling mixing index is not found much. Therefore, we suggest it is necessary to develop new non-sampling mixing indices.

\section{References}

[1] L. T. Fan, S. J. Chen, C. A. Watson, "Solids Mixing," Industrial Engineering Chemistry, vol. 62, no. 7, pp. 53-69, 1970.

[2] B. N. Asmar, P. A. Langston, A. J. Matchett, "A generalized mixing index in distinct element method simulation of vibrated particulate beds," Granular Matter, vol. 4, no. 3, pp. 129-138, 2002.

[3] M. S. Siraj, S. Radl, B. J. Glasser, J. G. Khinast, "Effect of blade angle and particle size on powder mixing performance in a rectangular box," Powder Technology, vol. 211, no. 1, pp. 100-113, 2011.

[4] M. Cho, "Particle-Mixing Simulations Using DEM and Comparison of the Performance of Mixing Indices," Trans. Korean Soc. Mech. Eng. B, vol. 41, no. 2, pp. 145-152, 2017. 